\title{
Testing whether drugs that weaken norepinephrine signaling prevent or treat various types of cancer
}

\author{
This article was published in the following Dove Press journal: \\ Clinical Epidemiology \\ 23 December 2009 \\ Number of times this article has been viewed
}

\section{Paul J Fitzgerald}

The Zanvyl Krieger Mind/Brain Institute, Solomon H. Snyder, Department of Neuroscience, Johns Hopkins University,

Baltimore, MD, USA
Correspondence: Paul J Fitzgerald National Institute on Alcohol Abuse and Alcoholism (NIAAA), 5625 Fishers Lane, Room 2N09, Bethesda, MD 20852-94II, USA Email pfitz@mbi.mb.jhu.edu

\begin{abstract}
Recently, I put forth the hypothesis that the signaling molecule, norepinephrine (NE), is an etiological factor in a number of types of cancer. In this brief commentary, I summarize evidence that NE plays a role in cancer and describe details involved in testing the hypothesis in humans through epidemiological investigation of existing medical records of persons who have taken pharmaceutical drugs that affect NE. If NE plays an etiological role in cancers of a number of organs, then taking a single pharmaceutical drug (such as clonidine, prazosin, or propranolol) that weakens NE signaling systemically, may simultaneously prevent or treat many different types of cancer, and this may represent a breakthrough in pharmaceutical prevention and possibly treatment of cancer.
\end{abstract}

Keywords: norepinephrine, acetylcholine, cancer, clonidine, prazosin, propranolol

In a recent paper, ${ }^{1}$ I put forth the hypothesis that the signaling molecule, norepinephrine $(\mathrm{NE})$, is an etiological factor in a number of types of cancer. This is an unusual hypothesis because NE is a neurotransmitter in the brain and sympathetic nervous system. Intracellular molecular pathways and hormones have been studied extensively in cancer research, but to my knowledge neurotransmitter signaling molecules have hardly been studied at all.

In this brief commentary, I summarize evidence that NE plays a role in cancer and describe details involved in testing the hypothesis in humans through epidemiological investigation of existing medical records of persons who have taken pharmaceutical drugs that affect NE. If NE plays an etiological role in cancers of a number of organs, then taking a single pharmaceutical drug that weakens NE signaling systemically may simultaneously prevent or treat many different types of cancer, and this may represent a breakthrough in pharmaceutical prevention and possibly treatment of cancer.

NE plays various signaling roles in many organs in addition to the brain. It is not a new concept that NE is involved in cancer as a group of Russian scientists and a group of Japanese scientists were already investigating the phenomenon in rodents several decades ago. ${ }^{2,3} \mathrm{I}$ put forth ${ }^{1}$ evidence that $\mathrm{NE}$ is an etiological factor in cancer related to both human and animal studies. I also pointed out that NE has direct access to many of the organs in which cancer can develop.

The most important point of my previous paper ${ }^{1}$ is that use of existing pharmaceutical drugs that either lower the level of NE (such as clonidine) or block NE receptors (such as propranolol and prazosin) may lower the probability of an individual developing cancer (and possibly treat existing cancers). Since the above three drugs, as well as 
plenty of similar drugs, have been on the market in the United States for decades, retrospective epidemiological analysis of the medical records of thousands (if not millions) of people who have taken these drugs is immediately feasible. This will be discussed below.

Why might NE affect cancer in the first place? Since NE is a sympathetic nervous system "stress hormone" that helps mediate the body's "fight or flight" response to environmental stressors, perhaps having (largely genetic) elevated NE tone keeps the body in a state of diminished maintenance or homeostatic processing, resulting in an increased probability of developing cancer. A related point is that boosting another signaling molecule, acetylcholine, with pharmaceutical drugs such as donepezil might help prevent cancer, since acetylcholine helps mediate the body's homeostatic, parasympathetic "rest and digest" functioning. In addition, the stress hormone epinephrine (adrenaline) may also play a role in cancer, as it activates the same endogenous adrenoceptors as NE, but there is less evidence that epinephrine plays such a role. It would also be informative to investigate whether the stress hormone, cortisol, affects cancer risk.

In testing the NE/cancer hypothesis epidemiologically, the basic idea is that pharmaceutical drugs that enhance NE signaling should increase the risk of cancer, whereas drugs that reduce NE signaling should decrease the risk of cancer. This latter possibility may seem counterintuitive, since most epidemiological studies to my knowledge have found that pharmaceutical drugs increase rather than decrease cancer risk. Regarding drugs that increase NE signaling: tricyclic antidepressants boost the level of NE (possibly systemically) and have already been associated with increased cancer risk, although this issue is controversial. ${ }^{47}$ Studying the effects of tricyclics on cancer risk, or two newer NE-boosting drugs, reboxetine and atomoxetine, is an area of research that would benefit significantly from greater investigation.

The most important avenue of epidemiological investigation into the NE/cancer hypothesis may be to study whether pharmaceutical drugs that weaken NE signaling lower cancer risk. These drugs comprise three main categories: alpha1 antagonists (such as prazosin), beta blockers (such as propranolol), and alpha2 agonists (such as clonidine). Drugs in the first two categories block postsynaptic NE signaling, whereas clonidine may principally lower the level of $\mathrm{NE}$ both centrally and peripherally, thereby decreasing NE transmission. All of these drugs have been on the market in the United States for decades and thousands of people have taken them and continue to take them, increasing the feasibility of epidemiological investigation. These drugs are typically used to treat hypertension or cardiac disorders. An important point is that when epidemiologically testing the effect of these drugs on cancer risk, it would be informative to compare persons on these drugs with persons who have the same disorder (such as hypertension) but are taking a drug that does not affect NE (such as a calcium-channel blocker) for the same condition. Comparison of cancer rates in persons on NE drugs with persons taking no pharmaceutical drugs at all would also be valuable. It would also be useful to not only test the effects of NE-weakening drugs on risk of a particular type of cancer, but also whether these drugs reduce cancer risk for a number of different cancers simultaneously. ${ }^{8}$ In addition, if there are enough data, it would be informative to test whether the treatment outcome of persons with existing cancers is affected by simultaneous use of NE-altering drugs.

A recent study indicates that exposure to doxazosin and terazosin, which are NE alpha1 receptor-blocking drugs, reduces the risk of prostate cancer, ${ }^{9}$ as does exposure to beta blockers. ${ }^{10}$ Exposure to beta blockers may produce a general decrease in cancer risk, ${ }^{8}$ which already provides evidence for a general effect of NE on cancer risk.

Acute boosting of NE probably has little or no effect on cancer risk, since the body's own fight-or-flight response releases large amounts of NE when activated, and people don't develop cancer each time this occurs (although repeated stressors may increase risk). Therefore, critical care patients who receive exogenous NE to maintain a stable hemodynamic status should not be at higher risk for developing cancer. I hypothesize that it is chronically elevated NE, or chronic use of NE-altering drugs, that significantly affects cancer risk. If so, pharmacoepidemiological studies that would examine the NE/cancer hypothesis should focus on individuals who are exposed to NE-altering drugs (or psychological stress) for months or years. Since drugs such as clonidine, propranolol, and prazosin are often taken for many years due to treatment of chronic hypertension or cardiac disorders, this increases the feasibility of epidemiological investigation of the hypothesis.

Which types of cancer might NE affect? One possibility is that NE affects cancer in every organ that it innervates. This includes the eye, brain, breast, spinal cord, heart, lung, blood, blood vessels, kidney, liver, stomach, pancreas, intestines, uterus, prostate gland, skin, and skeletal muscles. ${ }^{1}$ However, a counterpoint to the NE/cancer hypothesis is that both the heart and blood vessels, which have a high density of adrenoceptors, rarely develop tumors. Vascular malformations may be related more to growth factor phenomena than to adrenergic stimulation. On the other hand, a combination of 
effects by growth factors and adrenergic stimulation, perhaps at a cellular level following second messenger signaling, may well be a promising theory for carcinogenesis.

Even if chronic use of antidepressants or adrenergic receptor blocker medications does indeed have an effect on cancer risk, this does not confirm an etiological role for endogenous NE in cancer. It may merely indicate an association. Perhaps NE is a factor in the pathogenesis, and is a mediator somewhere in the much more complex process of developing cancer. In this scenario, use of NE-altering drugs may nonetheless be a means for lowering the probability of developing some types of cancer.

I would encourage epidemiologists reading this to strongly consider investigating the potential link between $\mathrm{NE}$ and cancer, as it could lead to immediate improvement in the pharmacological prevention and treatment of many types of cancer. Feel free to contact me for discussion.

\section{References}

1. Fitzgerald PJ. Is norepinephrine an etiological factor in some types of cancer? Int J Cancer. 2009;124:257-263.

2. Gurkalo VK, Zabezhinskii MA, Pliss GB, Krylov SS. Adrenergic component in the hepatotropic, carcinogenic effect of diethylnitrosamine. Biull Eksp Biol Med. 1977;83:455-458.
3. Tatsuta M, Iishi H, Baba M, Nakaizumi A, Ichii M, Taniguchi H. Promotion by bombesin of gastric carcinogenesis induced by N-methylN'-nitro-N-nitrosoguanidine in Wistar rats. Cancer Res. 1989;49: 5254-5257.

4. Sharp CR, Collet JP, Belzile E, Hanley JA, Boivin JF. The effects of tricyclic antidepressants on breast cancer risk. Br J Cancer. 2002;86: 92-97.

5. Sternbach H. Are antidepressants carcinogenic? A review of preclinical and clinical studies. J Clin Psychiatry. 2003;64:1153-1162.

6. Tamim H, Boivin JF, Hanley J, Stang M, Collet JP. Risk of breast cancer in association with exposure to two different groups of tricyclic antidepressants. Pharmacoepidemiol Drug Saf. 2006;15:689-697.

7. Tamim HM, Mahmud S, Hanley JA, Boivin JF, Stang MR, Collet JP Antidepressants and risk of prostate cancer: a nested case - control study. Prostate Cancer Prostatic Dis. 2008;11:53-60.

8. Algazi M, Plu-Bureau G, Flahault A, Dondon MG, Le MG. Could treatments with beta-blockers be associated with a reduction in cancer risk? Rev Epidemiol Sante Publique. 2004;52:53-65.

9. Harris AM, Warner BW, Wilson JM, et al. Effect of alpha1-adrenoceptor antagonist exposure on prostate cancer incidence: an observational cohort study. J Urol. 2007;178:2176-2180.

10. Perron L, Bairati I, Harel F, Meyer F. Antihypertensive drug use and the risk of prostate cancer (Canada). Cancer Causes Control. 2004;15:535-541.
Clinical Epidemiology

\section{Publish your work in this journal}

Clinical Epidemiology is an international, peer-reviewed, open access journal focusing on disease and drug epidemiology, identification of risk factors and screening procedures to develop optimal preventative initiatives and programs. Specific topics include: diagnosis, prognosis, treatment, screening, prevention, risk factor modification, systematic

Submit your manuscript here: http://www.dovepress.com/clinical-epidemiology-journal

\section{Dovepress}

reviews, risk \& safety of medical interventions, epidemiology \& biostatical methods, evaluation of guidelines, translational medicine, health policies \& economic evaluations. The manuscript management system is completely online and includes a very quick and fair peerreview system, which is all easy to use. 